\title{
NOTAS PARA EL ESTUDIO DE LA SECULARIZACIÓN DE LA CULTURA: DOS INICIATIVAS IRÉNICAS DE LEIBNIZ
}

\author{
POR \\ JAIME DE SALAS ${ }^{1}$ \\ Universidad Complutense de Madrid
}

\begin{abstract}
RESUMEN
El artículo se aproxima a dos iniciativas irénicas en las que Leibniz intervino en los años centrales de su carrera: la de Cristóbal de Rojas que buscaba la reconciliación de católicos y protestantes y la que Leibniz y Molano sostuvieron con D. E. Jablonsky dentro de las relaciones de las cortes luterana y calvinista de Hannover y Berlín. Estudia particularmente la dual dimensión desde la que interviene Leibniz: como miembro de la Corte de Hannover y como autor de una metareflexión que subraya la importancia moral de esta iniciativa, para la cual son de interés la distinción orteguiana entre ideas y creencias, así como la atención a la forma.
\end{abstract}

PALABRAS CLAVE: ecumenismo; Leibniz; Bossuet; diálogo; creencia; Ortega y Gasset; secularización; Habermas; Molano; D. E. Jablonsky; Religión y Política en el siglo XVII.

\section{TWO IRENICAL INITIATIVES OF G. W. LEIBNIZ. NOTES ON THE SECULARIZATION OF CULTURE}

\begin{abstract}
The paper studies two irenic initiatives in which Leibniz took part in the central years of his career. That of Cristóbal de Rojas which sought the reconciliation of catholics and protestants and that maintained with Molanus and D.E. Jablonsky involving the protestant courts of Hannover and Berlin. Special attention is paid to the dual aspect of Leibniz's position simultaneously following the official line of the dukedom and acting according to a moral vision of an unified humanity that led him to seek in practice new formulations of basic theological terms. We also attend to the dialogue form used by the participants.
\end{abstract}

KEY WORDS: Ecumenism; Leibniz; Bossuet; Dialogue; Belief; Ortega y Gasset; Secularisation; Habermas; Molanus; D. E. Jablonsky; Religion and Politics in the seventeenth century.

CómO CITAR ESTE ARTíCULO / CITATION: Salas, J. de 2018. "Notas para el estudio de la secularización de la cultura: dos iniciativas irénicas de Leibniz». Hispania Sacra 70, 141: 85-93. https://doi.org/10.3989/hs.2018.008

Recibido/Received 09-05-2017

Aceptado/Accepted 09-06-2017

INTRODUCCIÓN METODOLÓGICA

Para quienes nos acercamos al tema de la secularización de la cultura en la modernidad, la obra de Ortega es interesante aún cuando se elaboró antes de que la moderna discusión arrancara con La legitimidad de la edad moderna de Blumenberg. En cierta medida reproduce una actitud que de una forma lata podemos entender por nieztscheana que abunda en la necesidad y capacidad del individuo de crearse

1 j.desalas.ortueta@gmail.com / ORCID iD: http://orcid.org/00000002-7116-4091 su propia realidad. ${ }^{2}$ Aún más importante para el estudio del proceso de secularización es la distinción orteguiana de ideas y de creencias que apunta a una tensión permanente en una cultura propia de una sociedad histórica: el individuo debe adaptar su representación de la realidad a los distintos

2 Ortega y Gasset 2006-2009-2010: vol. 6, 373: «El hombre 'moderno' sustituye a la fe en Dios y en la razón». Es una forma sumaria para resumir un proceso que resulta mucho más complejo. Una visión más pormenorizada serían las lecciones de En torno a Galileo sobre todo el capítulo X de Ortega y Gasset 2006-2009-2010: vol. 6, 469, que no llega a tener en cuenta la situación del siglo XVII donde Descartes obtiene demasiado protagonismo. 
cambios y exigencias que la sociedad va imponiendo. El paso de creencias a crisis, y de crisis a ideas, y la consolidación de nuevas formas de vigencia, es decir de nuevas creencias, aparece a la vez como la expresión de la permanente fragilidad de la cultura efectiva de Occidente $y$, por otra parte, su mayor triunfo. ${ }^{3}$

Esta distinción orteguiana constituye uno de los recursos más importantes para la comprensión de las culturas de una determinada época, si bien hay que complementarla teniendo en cuenta el carácter dinámico que el pensamiento adquiere cuando se la entiende dentro de su coyuntura. El trabajo de la inteligencia consiste en buscar formas de equilibrio que permiten la vida efectiva en una época, pero de hecho se desarrolla dentro de un acontecer cambiante al que hay que adaptarse y dar respuesta. En realidad, lo decisivo no son propiamente las representaciones que guían los actos sino la incidencia de estas creencias e incluso de las ideas - que pueden adquirir una dimensión creencial- en el comportamiento. Para ello hay que tener en cuenta la perspectiva del propio agente. En este sentido, Ortega entiende que las creencias se hacen presentes en el desarrollo de la vida individual, en la acción misma en que consiste el vivir. ${ }^{4}$

¿Cómo se aplican estos conceptos al estudio del problema de la secularización? En la práctica la distinción entre ideas y creencias se vuelve problemática: cuando se quiere aplicar a situaciones concretas resulta muy arduo encontrar claramente a lo que se refiere. Por ejemplo, si entendemos por creencia, la creencia en Dios. Por un lado, la creencia en Dios se flexiona en una multiplicidad de creencias distintas, relativas a la revelación en que se apoya la misma religión, los dogmas y teorías que mantiene, la representación de la relación del creyente con la divinidad, el papel de la misma Iglesia, la concepción del tiempo, la comprensión del universo y el papel que el creyente debe asumir en él, etc. Algunas de estas creencias pueden ser inconscientes; otras conscientes, pero no tematizadas, sino consagradas por el uso. Por otro lado, los motivos religiosos muestran una extraordinaria capacidad de aglutinarse con prácticamente todas las formas culturales de la vida cotidiana. Están presentes en rituales familiares y sociales, en prácticas profesionales y en hábitos de trabajo, y en experiencias de todo tipo que tienen otras dimensiones en principio más importantes. Al tiempo, muchas de estas creencias son sustituibles por otras o por lo menos admiten redefinición en situaciones sociales nuevas formando nuevas constelaciones de creencias. Y esto acontece en el contexto general de la gestación de una cultura profana que genera sus propios puntos de referencia. Por ello también tiene razón Ortega cuando mantiene que la racionalidad que las creencias dan a la vida no se relaciona con un sistemátismo de las propias creencias, sino que se logra desde la misma perspectiva vital y concreta del individuo. ${ }^{5}$

\footnotetext{
3 Ortega y Gasset 2006-2009-2010: vol. 6, 571.

4 Ibídem: 48 y por el contrario tiende, a mi juicio, a minusvalorar el alcance que costumbres e instituciones tienen en la constitución de la misma individualidad: por ejemplo en el análisis del uso, ejemplificado en el saludo. Ibídem: vol. 10, 266. Incluso entiendo que habría que establecer importantes matizaciones en lo que respecta a la contraposición entre usos y creencias.

5 Ortega y Gasset 2006-2009-2010: vol. 9, 176 y ss.
}

Hay una segunda dificultad relativa añadida al estudio a posteriori del hecho histórico, más que al comportamiento de los agentes. Efectivamente, el acto político es un acto de comunicación que no sólo transmite unas decisiones, sino que se da dentro de un contexto previo de interlocución. Estudiar esta interlocución se ve enfrentado con una doble dificultad. Por ejemplo, Leibniz y Molano estaban en constante contacto, pero de una forma oral sin que haya quedado un rastro que nos enseñe como se influyeron mutuamente. La poca correspondencia no resulta muy explícita en este sentido. Se podía decir lo mismo de las relaciones con la duquesa Sofia, la gran valedora de Leibniz. Sólo nos hacemos idea de la relación y del mutuo influjo en cuestiones de la corte con la correspondencia. ${ }^{6} Y$ esta dificultad se extiende a la inserción dentro de la Corte de Hannover donde la comunicación sería fundamentalmente oral. Justamente porque se trata de una cuestión que remite a un estado de opinión, de los gobernantes y de sus asesores, la falta de conocimiento de la comunicación oral constituye un impedimento importante. En la medida en que una de las funciones de la correspondencia era suplir esa falta de presencialidad, paradójicamente podemos acceder mejor a la realidad de unos intercambios que hoy serían sencillamente supuestos en función de las decisiones que se tomaron o de la noticia de las actividades de los distintos agentes. Alcanzamos a saber más de lo que pensaban que si no hubiera correspondencia, pero ésta no constituye sino una parte del contexto en el que se tomaron decisiones.

A esto se añade una observación del propio Ortega por la que "todo decir es deficiente» en la medida en que la comunicación oral o escrita hace referencia a un estado de cosas en la que se encuentran insertos los interlocutores y que nosotros podemos tener dificultad de comprender aún cuando poseamos el conocimiento del idioma en el que se hace la comunicación. ${ }^{7}$ Hay un trasfondo de creencias que los interlocutores no dicen porque no necesitan decir y que sin embargo constituyen el contexto en el que la comunicación es efectiva. ${ }^{8}$

Finalmente hay que subrayar que se busca llegar a un acuerdo práctico. Entre los modos de comunicación posibles, la ciencia y la filosofía representan un extremo en su grado de elaboración y su dependencia en un nivel que refleja la misma historia de la disciplina, pero en las cuestiones políticas -independientemente de la importancia de la historia de las ideas - tenemos que hacer frente a una opinión pública en un contexto determinado. No se trata de la opinión pública que se abre paso en el mundo occidental

6 En este caso, puede servirnos de ejemplo una carta enviada por la duquesa a Leibniz durante la estancia de éste en Viena en 1690. En ella, se muestra no sólo completamente favorable a la iniciativa leibniziana, sino involucrada personalmente en la misma - a través de Leibniz-, pero también a través de su hermana Luisa Hollandine abadesa del Convento de Maubuisson: «la iniciativa del Obispo de Tina (Cristóbal de Rojas) es también digno de elogio y sus razonamientos admirables... hay que esperar en este tema una revelación extraordinaria y de la misma manera que el cristianismo llego a este mundo por una mujer, sería para mi un hecho glorioso, que se hiciera por mi, pero hace falta influencias particulares para lograrlo». Leibniz 1923: 1923 y ss.

7 Ortega y Gasset 2006-2009-2010: vol. 6, 213.

8 Ibídem: vol. 9, 9-733. Entiendo que las creencias constituyen, al menos en parte, aquello que no está dicho pero aceptado como parte de la realidad. 
a partir del siglo XVIII. Es, en rigor, la opinión de unos pocos sobre cuestiones comunes, personas que se encuentran mayormente agrupados en torno a la Corte, en este caso de Hannover, pero que tienen que avalar una decisión como la apropiada. Por ello, se trata de un discurso que se desarrolla atento a la coyuntura como a la dinámica del ejercicio del poder y en este discurso es necesario encontrar un acuerdo que sea funcional en el sentido que tenga en cuenta las realidades que cualquier discurso político ha de tener en cuenta. En el caso de una negociación irénica el acuerdo tiene que ser doble en cuanto que una parte debe poder actuar en nombre de quiénes representa y por otra ha de lograr el asentimiento del contrario. La política imprime un cariz a la sociedad, pero al tiempo depende directamente de ella a la que el curso de la historia le sobrevienen fenómenos inesperados que tienen dimensiones positivas y negativas.

La dificultad de utilizar la categoría de secularización remite a la complejidad del fenómeno mismo. Es un fenómeno de crisis y de debilitamiento de creencias, pero dentro de eso veremos que las conversaciones en las que Leibniz interviene ofrecen tres niveles distintos: la de la experiencia de la fe del creyente; la de la teología vigente y la de la historia de la Iglesia a la que remiten los cristianos con distintas interpretaciones, pero en realidad desde una matriz común en el que todavía el hombre europeo se siente insertado; y finalmente la posición de la Iglesia como institución que apoya la práctica de la religión dentro de una sociedad en la que el Estado en gran medida ha pasado a intervenir y mediatizar directamente en su gestión. Las conversaciones irénicas que comentamos, van a mostrar que estos tres niveles siguen dinámicas distintas, aunque se encuentren interrelacionados.

Así, en la medida en que la Reforma es una cuestión de experiencia religiosa, parece que tendríamos que ver en ella más una radicalización de la experiencia previa que, lejos de desaparecer, adquirió formas nuevas. Más, en la medida en que la diferencia afecta a la institucionalización de la fe, la Reforma implica una crisis de instituciones. Podemos contraponer la incertidumbre ante la salvación de algunas doctrinas protestantes, por ejemplo al debilitamiento de la Iglesia católica como institución universal. Por ello, la crisis se resolverá no sólo con la Contrarreforma que implicó nuevas formas de sensibilidad religiosa, sino en general con el debilitamiento de la autonomía y radio de acción de la Iglesia católica como institución universal, y la aparición de iglesias nacionales tanto en el mundo protestante como en el mundo católico, vinculadas al poder real. La Iglesia católica no sólo es excluida formalmente de ámbitos protestantes, sino también tiene que aceptar, en términos generales, un papel secundario en la nueva institucionalización de la política que acontece a lo largo del siglo XVI hasta el XVIII en el mundo europeo, a pesar de que la religión retenga un valor simbólico claro en la vida cotidiana de la mayor parte de la población.

BREVE NARRACIÓN DEL DESARROLLO DE LAS CONVERSACIONES

Los dos episodios que ocupan este trabajo están relacionados con el proceso de secularización cultural en toda su complejidad. Son dos negociaciones irénicas en las que Leibniz intervino directamente. La primera iniciada por el obispo Cristóbal de Rojas y Spinola buscaba la reconciliación de los católicos con los protestantes. Mientras que el segundo apuntaba a una reconciliación interna de los protestantes, buscando el entendimiento entre luteranos y calvinistas. Sin embargo son muy distintos si se pretende entenderlas como actos unitarios que se suceden a lo largo de un periodo dilatado de tiempo.

Efectivamente en el primer caso la iniciativa de Rojas representando al Vaticano y al tiempo al Emperador, comienza en 1675 al entrar en contacto con varios estados protestantes del Imperio con el fin de iniciar contactos con las confesiones con vistas a superar la distancia que la Reforma había supuesto (Sajonía, Brandenburgo, el Palatinado entre los más importantes). Por ello las conversaciones que se realizaron en Hannover y que afectaron a Leibniz, no serían más que una de las diversas aproximaciones que Rojas intentó. Pero fue probablemente aquélla en la que se llegó más lejos dejando aparte la experiencia previa de Rojas en los territorios húngaros de los Habsburgos. En primer lugar, pasó por Hannover en 1676 donde es recibido por Juan Federico, el duque que empléo a Leibniz. La iniciativa fue bien acogida en la Corte incluso después de la muerte de Juan Federico en 1679. El contacto de Rojas fue Molano, el director de asuntos eclesiásticos en la Corte, y no parece que interviene Leibniz directamente. ${ }^{9}$ En este primer momento de las negociaciones entre el papa y el ducado de Hannover hay un momento decisivo en 1683. En este año Rojas escribe Regulae circa Christianorum omnium ecclesiasticam reunionem... y Molano, abad del monasterio de Loccum, responde con su Methodus reducendae Unionis Ecclesiaticae inter Roamenenses et Protestantes ex speciali mandato Serenissimi Principis ac Domini... El escrito de Rojas dio lugar a una ardua polémica. ${ }^{10}$ Dicha reacción significó un contratiempo para la iniciativa en el contexto del Imperio en general. Sin embargo, el duque Ernesto Augusto tenía la intención de buscar un acercamiento al Imperio para convertir el ducado de Hannover en el noveno elector del Imperio. Por ello, las conversaciones irénicas resultan oportunas en un momento en que el ducado esta prestando apoyo al Imperio en su lucha con los turcos. La gravedad de la coyuntura política se refleja en el hecho de que a la sazón Viena estaba siendo sitiada por los turcos y el emperador necesitaba este apoyo. La lucha contra el turco adquirió la dimensión de una cruzada.

En este primer momento, no se estableció un intercambio epistolar significativo, pero es muy posible que en la larga temporada que Rojas pasó en Hannover en 1683 conociera y tratara al pensador alemán. La redacción del Systema Theologicum por parte de Leibniz, probablemente de $1686^{11}$, se realizó pensando en esta situación donde el primer paso desde el punto de vista de la Corte de Hannover la había dado Molano, y coincidía con una política favorable a la autoridad imperial. El hecho es que la intervención de Leibniz que conocemos por su correspondencia, se realiza tardíamente y por ello el trabajo de Masser sobre Molano y Rojas constituye una adición importante, mientras que nos

9 Miller y Spielman 1962: 33 y ss. De todas formas es prácticamente seguro que Leibniz conocería estas relaciones. Para una visión de conjunto de las relaciones irénicas, cfr. Neveux 1968: 69 y ss.

10 Miller y Spielman 1962: 49 y ss.

11 Es el mismo año en el que Leibniz redacta el Discurso de Metafísica y entra en correspondencia con Arnaud. 
quedan trabajos significativos que ayuden a valorar mejor la intervención de Bossuet.

Por otra parte, hay una importante segunda parte a estos intercambios irénicos que comienza en 1690 donde Leibniz interviene de una manera más importante dejando ahora en segundo lugar a Molano. Aquí se puede acudir a la correspondencia que ha sido recogida en los volúmenes de obras de Leibniz de Foucher de Careil, Onno Klopp y la Academia de Ciencias de Berlin. Son significativos los trabajos que Leibniz realiza para comenzar un diálogo con Pellison, autor de Reflexions sur les differends de la Religion y a partir de este punto pronto comenzó la correspondencia con Bossuet. En la intervención de Leibniz pesa la mediación de Madame de Brinon afincada en el convento de Maubuisson, donde la abadesa Luisa Holandine era la hermana de la duquesa Sofia. Pero Molano sigue estando presente en la medida en que es el autor de los textos que deben ser aprobados o contestados por la parte católica y que constituyen el punto de referencia de las conversaciones. Así, redactó Cogitationes privatae para Bossuet que Leibniz manda al prelado francés en diciembre $1691 .^{12}$ A ello se añade otro texto De scripto cui titulus Cogitationes Privatae y un complemento de las Cogitationes, que se terminan el 18 de julio de 1694. Por su parte, Leibniz interviene con escritos como Des Méthodes de Reunión, Reponse de Leibniz au mémoire de l'abbé Pirot ${ }^{13}$, Projet au nom de l'abbé de Lockum pour faciliter la reunión des protestants avec les romains catholiques, y finalmente Observations sur l'écrit de Monsieur l'evèque de Meaux, ya de 1702, pero la superioridad institucional de Molano era clara. Era su terreno y Leibniz intervenía haciendo valer su competencia y erudición, pero también su amistad y el apoyo de la duquesa Sofia.

En realidad, tenemos más medios para fijar la situación de comunicación en este segundo período entre 1690 y 1695 , año en el que muere Rojas. Madame de Brinon no sólo se dirige a Leibniz, sino que se prestó a actuar de intermediaria con Pellison y con Bossuet y con ello comienza una interlocución de extraordinario interés. Para su estudio no es suficiente la importante aportación que están haciendo los volúmenes de las Obras completas, editadas por la Academia que incluyen cartas de y a Leibniz, sino que son significativas otras correspondencias como las que mantiene Bossuet con Pellison o Madame de Brinon, o incluso las cartas de la duquesa Sofia con su hermana. Ello permite a integrar distintos puntos de vista. De esta forma mantienen su valor los volúmenes antiguos de Foucher de Careil, aunque sea comprensible que la edición de la Academia sacrifique la variedad de interlocutores, restringiéndose a las cartas a Leibniz y las respuestas de éste, incluyendo sus escritos teóricos pertinentes.

Se da en estas conversaciones una tercera fase a partir de 1698. Foucher de Careil ha publicado un escrito de Leibniz, dirigido al Conde Buchaim en el que se relata la visita del nuevo obispo de Neustadt a la Universidad de Helmstedt. ${ }^{14}$

\footnotetext{
12 Bossuet 1848: 16-583.
}

13 Ibídem: 17-151.

14 A 1-6-20. Leibniz redactó este informe con la intención explicita (A 1-6-19) de que el duque Antón Ulrich lo utilizara como complemento a una carta, también redactada por el propio Leibniz, que el duque envío a Luis XIV (A 1-6-26).
Por otra parte, la interlocución con Bossuet se vuelve a abrir después de varios años de silencio a través de la mediación del Conde de Heron, representante de la corona francesa en la Corte de Wolfenbüttel. Se mantiene así la antigua vía de comunicación a través de la duquesa Sofia y su hermana Luisa Hollandina, abadesa de Maubuisson con la intervención de Madame de Brinon. Sin embargo, es el duque Antón, Ulrich de Wolfenbuttel, quién ahora se presenta cómo el apoyo político de primer orden cara a la negociación.

El otro gran intento, mucho más circunscrito en lo temporal, es la intervención de Leibniz en una negociación para la reconciliación entre calvinistas y luteranos. ${ }^{15}$ También en este caso se dieron unos condicionantes políticos importantes en la medida en que se trata de una iniciativa de dos Coronas protestantes. La una luterana-Hannover mientras que la otra- Brandenburgo-calvinista. El año en que comienza la negociación, 1697, es también el año de la Paz de Ryswick, lesiva para la causa protestante y también, el año de la conversión del elector Augusto el fuerte de Sajonia al catolicismo, que debilitaba la causa del protestantismo en Alemania. La negociación también se apoyaba en la boda real entre Sofia Carlota, cuñada de Jorge Luis el elector en 1697 de Hannover, y Federico I de Brandenburgo.

Como en casos anteriores la negociación se centra en unos textos fundamentales que dan pie a réplicas y aclaraciones aparte de las cartas que los interlocutores mantienen entre ellos. El texto inicial es de D. E. Jablonsky Kurze Vorstellung der Einigkeit und des Unterscheides im Glauben, beyder Evangelischen so genandten Lutherischen und Reformiten Kirchen. Al que contestó Leibniz en el año siguiente con Tentamen expositionis irenicae trium potissimarum inter protestantes controversiarum ${ }^{16}$ y eventualmente con Unvorgeifliche Gedanken über eine Schrift gesandt kurze Vorstellung. ${ }^{17}$

Rösler explica que existiría un origen remoto de la negociación en las conversaciones entre el duque Antón Ulrich de Wolfenbuttel y Federico Guillermo de Brandenburgo en 1697. Pero el comienzo oficial sería la entrega por parte del embajador de Prusia, Ezequiel von Spanheim, de la Kurze Vorstellung en la Corte de Hannover.

\section{LA DIMENSIÓN POLÍTICA DE LAS CONVERSACIONES}

En términos generales el origen de estas negociaciones es fundamentalmente político en la medida en que la iniciativa inmediata responde a intereses políticos de algunos de los interlocutores: Francisco de Rojas es enviado por el papa y el Emperador por común acuerdo ante la amenaza de los turcos en la frontera este del Imperio ${ }^{18}$; el duque Ernesto Augusto desea lograr el noveno electorado

15 En este caso nos apoyamos en la extraordinaria aportación de Jablonski y Leibniz 2013.

16 A 1-7-371.

17 A 1-7-424 y ss. da una primera versión de un texto que recibe varias versiones hasta recibir su versión definitiva en 1704 . Sería, de acuerdo con Rösler, la posición madura de Leibniz contrastando en ese sentido con el arriba citado Tentamen, que por su parte representa un estadio intermedio en el pensamiento de Leibniz.

18 Se trataba de lograr en el Imperio el espíritu de cruzada que había dado pie a la victoria de los aliados contra los turcos en Lepanto, en 1571. 
para Hannover, o el hecho de que las Cortes de Hannover y Berlín encuentran que ésta es una iniciativa provechosa para ambas partes cuando las Coronas protestantes se encuentran amenazadas por el éxito de Luis XIV.

El término de las negociaciones es por fuerza mayor proviniendo normalmente de la muerte de uno de los interlocutores o de cambios en la coyuntura política: Las muertes de Pellison, Bossuet, o Rojas vienen a marcar etapas. Tambien hay que tener en cuenta la promulgación del Act of Settlement de 1701 por el parlamento británico que nombra a la duquesa Sofia heredera de la Corona inglesa en el caso de no tener descendencia la reina Ana. ${ }^{19}$ Esto determina, a pesar de que Leibniz siga implicado en las dos negociaciones al mismo tiempo, una creciente importancia de la sucesión de la Corona inglesa en la Corte de Hannover. En el caso de la negociación entre Hannover y Berlín tanto Jablonsky como Leibniz y Molano recibieron de sus respectivas Cortes la instrucción de no intervenir en la discusión sobre la manera en que la reina de Prusia debía de recibir la comunión. Rösler apunta que en este punto la misma coyuntura política que había facilitado un entendimiento pasa a impedirlo. Desde el punto de vista de las creencias, lo que más se debe resaltar es el peso de la política nacional en una cuestión fundamentalmente religiosa. En estos casos el estado va heredando la estructuración de una sociedad organizada culturalmente en el ambito de la importantísima variable religiosa. El aumento progresivo de poder del estado más que la falta de unión de los cristianos en la Iglesia católica parece la respuesta estructural a una crisis de instituciones. En cambio, a nivel popular las prestaciones de la religión siguen intactas hasta después de la revolución industrial y el gran éxodo del campo a la ciudad.

Sin embargo, a nivel de experiencia religiosa la cuestión es muy distinta. En este caso no pensamos en la institucionalización de la religión ni en su vigencia popular sino las formas de interlocución de quiénes formaban parte de una minoría de hombres cultos que intervenían como expertos o funcionarios. Se da un contraste fuerte entre el papel relativamente secundario que muchos de estos tenían en la decisiones políticas, pero al tiempo el compromiso con la reconciliación de las Iglesias que algunos de ellos llegan a mostrar. En el caso de Leibniz, el paso a buscar el apoyo del duque Antón Ulrich - que todavía no ha sido estudiado con detalle- es muy expresivo. Leibniz sabe que tiene que ajustarse a los tiempos y exigencias de un juego, pero por otro lado, ese mismo juego le permite tomar iniciativas. El caso de Jablonsky y de Molano también apunta a personas que actuaban movidos por un principio que tenía desde luego implicaciones políticas conocidas y asumidas por ellas mismas desde unas convicciones irénicas.

En el caso de Leibniz podemos apreciar que la preocupación por la unidad de las Iglesias esta en el comienzo de la carrera y es una constante de la misma. Así podemos pensar en su gestión en Maguncia con el barón de Boineburgo. ${ }^{20} \mathrm{La}$ figura de Molano se debe entender como el resultado de una orientación intelectual que remite a la figura de Georg Calixto y la Facultad de Helmstedt, y de hecho fue discutido a lo lar-

19 El hijo de la Duquesa pasará a ser, además, de duque de Hannover, el rey Jorge I en 1714.

20 Duchhardt 2015: 51. Cfr. asimismo Jablonski y Leibniz 2013: 19. go de su trayectoria desde posiciones luteranas rigoristas. ${ }^{21}$ La figura de Jablonsky tiene una dimensión distinta porque su preocupación fundamental y en cierto medida excluyente era el de la unidad de los protestantes por haber sido víctima de persecución por parte de los católicos. No es posible comprender las negociaciones sin tener en cuenta que estos tres participantes aunque estaban subordinados a sus respectivas autoridades, se movían por un sentimiento religioso que en términos del siglo siguiente puede entenderse como amor a la humanidad. En este sentido, la oportunidad política que da paso a estas negociaciones no debe ocultar una autentica ética en la que nos debemos detener. Se trata de unas ideas reformulación de creencias anteriores que se empiezan a alumbrar en iniciativas de esta naturaleza y que adquieren desde el principio una dimensión creencial por ser peso en el juego de la política del momento.

\section{LA FIGURA DE LEIBNIZ EN EL CONTEXTO DE LA DISTINCIÓN ENTRE IDEAS Y CREENCIAS}

¿En que sentido se puede hablar de una transición de creencias a ideas? ¿En que medida nos encontramos ante un proceso de secularización? Volvamos a la distinción ya hecha en el epígrafe II. En un sentido la secularización de la sociedad como perdida de la fe no se puede observar, sino solo una cierta transferencia de poder de las Iglesias a los estados que las apoyan; En cambio, en lo que respecta a trasfondo de creencias religiosas relativas a la historia de la Iglesia, o a la teología, la cuestión es distinta: Así las conversaciones se realizaron en un contexto donde las discusiones del dogma, de la liturgia y del régimen sacramental, de libros simbólicos y de las fuentes de la revelación eran comunes y bien conocidas, aunque abiertas muchas veces a discusión dentro de foros restringidos. Pero ahora las creencias de antaño y sus sistematizaciones tienen que encontrar encaje en un mundo donde las realidades políticas y culturales están cambiando. El mundo europeo se encontraba en un momento de extrema vitalidad con la aparición de nuevas formas de experiencia religiosa: janseinismo, quietismo, pietismo, el irenismo de la escuela de Helmstedt, el socianismo, la extraordinaria presencia educativa de la orden de Jesús, donde la religiosidad leibniziana, en principio, destaca sobre todo por el intento de síntesis con la experiencia científica de la ciencia postgalileana. ${ }^{22}$

Así pues, resulta importante resaltar que, si bien no se llego a ningún acuerdo sobre todo por razones propiamente políticas, había un sustrato de creencias comunes relativas al papel de la Iglesia en la sociedad, a la importancia de la historia de los concilios para definir las posiciones dogmáticas, a la conveniencia de respetar la configuración de la institución independientemente de que la reforma había relativizado su peso político, que permitían e incluso aconsejaban las negociaciones y sobre este fondo se inicia una época especialmente creativa en lo que respecta al

\footnotetext{
21 Ibídem: 861. Calixto murió en 1656, pero su hijo continua su trabajo.

22 De todas formas, últimamente se da un proceso de valoración de extraordinario conocimiento por parte de Leibniz de la teología tradicional por parte de la Academia. Cfr. Antognazza 2007.
} 
pensamiento religioso. Pero al tiempo se daba una gran dificultad para lograr un consenso, primero porque la deriva de la cultura del siglo conducía a una forma de naturalismo que representa la obra de Locke, por ejemplo, y además porque el mismo poder político que permitiría las conversaciones en una determinada coyuntura, se podría mostrar renuente en otro momento.

La figura de Leibniz resulta especialmente importante, justamente por el equilibrio que encuentra entre distintas facetas de su actividad intelectual. Como político su acción tiene lugar en una coyuntura concreta y se despliega desde la situación de una persona al servicio de la Casa de Hannover. Pertenece a un tercer nivel de personas que intervienen en los asuntos políticos, asesoran pero propiamente no toman decisiones por oposición a elector o duque y a sus ministros principales. ${ }^{23}$ Sería en ese sentido un técnico. Pero al tiempo su compromiso con la causa de la reconciliación de las Iglesias, como hemos visto, es una constante de su actividad intelectual y por ello debemos ver en su intervención en estas iniciativas no sólo comportamiento político oportuno, sino el anticipo de una aspiración ilustrada de una humanidad, además de la conciencia de la fragilidad de un orden que con la Reforma pierde un elemento importante de cohesión la unidad de la religión. Ciertamente la debilidad del Imperio en los años en torno a 1690 aconsejan su intervención tardía en las negociaciones que Cristóbal de Rojas inició, mientras que la preponderancia de la hegemonía francesa a partir de la paz de Ryswick e inminencia de la Guerra de Sucesión española implica un contexto en el que la condición de protestante facilitaba un frente anti-francés dentro y fuera del Imperio. Pero en estas figuras se da una orientación humanitaria fundamental y de ahí una búsqueda en el caso de Leibniz de una reformulación de una cultura en la que por otra parte se encontraban. Se puede aplicar la alternativa ideas y creencias, en la medida en que ante la crisis de unas creencias establecidas se da, no tanto la gestación directa de una nueva idea que pueda sustituir las creencias que han perdido su vigencia, sino una reformulación de ésta que adquiere nuevos usos, al tiempo que su entorno se transforma sin contradecir explícitamente el uso que ha tenido hasta el momento. En este contexto se debe entender la idea de la humanidad que desde San Agustín se agrupa en la Ciudad de Dios de acuerdo con el contexto de la revelación, por encima de la diferencia de etnias y de culturas, como beneficiada de una común revelación. El pensamiento de Leibniz gira en torno a la catolicidad de las religiones cristianas. Dice a Madame de Brinon la mediadora católica en el intercambio con Bossuet:

Tiene Vd. razón de juzgarme católico en el corazón. Incluso lo soy abiertamente: pues solo la obstinación hace al hereje, y es algo que gracias a Dios, que mi conciencia no me reprocha. La esencia de la catolicidad no es el comulgar externamente con Roma[...] La comunión verdadera y esencial, que hace que seamos del cuerpo de Jesucristo, es la caridad[...] aquellos que están dispuestos a hacer todo lo que incluso para mantener la comunión externa, son católicos de hecho. ${ }^{24}$

\footnotetext{
23 Jablonski y Leibniz 2013: 23.

24 Leibniz (A) 1-6-235.
}

Ello conduce a una visión secularizada de la historia, como producto de un progreso inmanente, causado por el propio desarrollo de las mónadas en camino a una realidad más perfecta. ${ }^{25}$

¿Cual es el contenido de este entendimiento ideal? Es notable y particular al genio leibniziano que reflejando la voluntad de reparar la escisión que la Reforma había abierto, su proceder en la práctica va a consistir en abundar en la razón apelando por encima de las peculiaridades del lugar y del momento: "la razón es la voz natural de Dios». ${ }^{26}$ En ese sentido, aunque mantenga el perfil de un teólogo que admite la revelación cristiana, sin embargo es consciente de la posibilidad de mejorar la suerte de la humanidad con la acción política ${ }^{27}$, sobre todo la del Príncipe. Por ello, «la ley de la naturaleza es la ley católica ${ }^{28}$ que la acción política, sobre todo del Príncipe ${ }^{29}$, en la mejor de sus expresiones debe seguir. Hay una acción de transformación de la naturaleza que hace las veces de religión y que el Príncipe debe aprovechar, que se deriva del uso conjunto de la razón para mejorar la realidad..$^{30}$

De esta manera habría que entender que, por encima de su cometido como administrador afecto a una corte, por razón de su extenso ámbito de contactos y el favor de la duquesa Sofia, Leibniz desarrollo un proyecto intelectual que incluía el uso del poder del Principe como colaborador del Dios creador. $Y$ de esta manera la oportunidad que gobierna la vida política se realiza dentro del marco de una praxis personal únicamente atribuible a este autor. ${ }^{31}$

Acercándonos más al tema de la conciliación de distintas religiones, está muy presente la convicción de que en el mejor de los mundos posibles, cabe encontrar una solución a las diferencias entre religiones. De hecho las soluciones desde el punto de vista teórico no son siempre satisfactorias en virtud de la contingencia de lo creado. Han de enfrentarse con obstáculos que, en el contexto concreto inmediato, pueden resultar insalvables. Así pues una actuación gobernada por el uso de la razón y la tesis de que este sería el mejor de los mundos posibles, tiene que ajustarse a las limitaciones de hecho. En este contexto, Leibniz enumera tres formas de resolver los problemas concretos ante los que se encuentran los negociadores ${ }^{32}$ : hay problemas que se pueden superar por un análisis conceptual que ponga de relieve que las diferencias se pueden superar mediante una determinada fórmula; en segundo lugar, hay cuestiones en los que cada parte puede ceder a la otra; en tercer lugar hay cuestiones que se deben dejar en suspenso en la espera de

\footnotetext{
25 Gilson 2005: 233.
}

26 Leibniz 1948: 139. Naert 1968: 101, hace un comentario de este pasaje.

27 Leibniz (A) 4-1-534.

28 Leibniz 1948: 49.

29 Leibniz (A) 6-4-2281.

30 De ahí la importancia de las Academias Reales. Fue Leibniz el primer presidente de la Academia de Ciencias de Berlín, y además intervino en los proyectos de otras.

31 Una primera aproximación por mi parte aparece en Derecho Natural y Coyuntura Política en Leibniz, intervención todavía inédita en el Congreso en Padua dedicado a Leibniz e il Diritto Naturale el 16 de Diciembre de 2016.

32 Leibniz (A) 1-5-10. Es un texto dirigido al Conde de Hessen Rheinfels donde sobre todo se detiene ante la eventualidad de un nuevo concilio. 
un concilio que las decida. Desde el punto de vista filosófico, es la primera forma la más importante en la medida en que se trata de encontrar fórmulas que superen efectivamente un aparente impasse. $Y$ es cierto que como ha mostrado Irena Backus con motivo de la negociación entre luteranos y calvinistas es perfectamente posible lograr esas fórmulas en cuestiones concretas. ${ }^{33}$ Puede que la misma capacidad intelectual de Leibniz determinara que sus interlocutores no fueran capaces de seguirle. Así ante Bossuet el esfuerzo de Leibniz de superar la mecánica cartesiana para ayudar a comprender el sacramento de la comunión, más bien era una dificultad añadida que una solución.

En lo que respecta a la concesiones mutuas, es un ámbito en el que los interlocutores pueden llegar a acuerdos, pero con ello se tiende a minusvalorar aquellos aspectos sobre los que se decide. En cualquier caso, lo que se impondría es una razón que pudiéramos llamar política por encima de lo que se puede llegar humanamente con una reflexión filosófica. Finalmente, este fue el gran escollo de las conversaciones con Bossuet en lo que respecta a un posible concilio al que acudirían tanto los católicos como aquellos protestantes que hubieran pactado. En la intención de Leibniz se encontraba la voluntad de que este nuevo concilio reuniera características que no había tenido el Concilio de Trento en lo que respecta a la asistencia de obispos de Francia, y sobre todo, representantes del bando protestante. $Y$ estas pretensiones eran mucho más de lo que el bando católico estaba dispuesto a aceptar. La oposición a la Reforma se hace también desde la valoración de la propia institución de la Iglesia católica como el camino hacia la salvación. Leibniz entenderá que en un concilio futuro, convocado de acuerdo con unas exigencias formales, se puede llegar a un acuerdo y ello para Bossuet equivalía a no aceptar la validez del Concilio de Trento y la valoración de lo que el mismo concilio representa dentro de la vida de la Iglesia.

En cualquier caso, esto significa que la negociación apunta a otro tipo de acuerdo que aquél con el que se acoge a una conclusión logicamente válida. Este tipo de acuerdo respondía a una de las intuiciones políticas más desarrolladas por el propio Leibniz, que tiene una dimensión creencial: la ventaja de la colaboración, sobre todo de la colaboración institucional entre países y entidades. Era una decisión razonable que podría dar paso a una colaboración fructífera. La decisión de colaborar por un bien común supera lo que para Leibniz podría entenderse como un inconveniente, el acto de la nuda voluntad de un agente que en realidad no está en condiciones de prever un futuro. La razón es algo que no sólo es beneficiosa a nivel particular, sino también lo es a nivel colectivo. Podría, aunque no fuera característico de un protestante, haber recordado la importancia de Constantino en el establecimiento de la Iglesia católica como la Iglesia del Imperio. ${ }^{34}$

\section{LIMITACIONES DE ALGUNOS DE LOS CONCEPTOS METODOLÓGICOS UTILIZADOS}

¿Como hay que valorar este diálogo? Podemos empezar por lo que realmente consiguió en términos políticos. De una

\footnotetext{
33 Backus 2016: 31

34 Kelly 2009: 21.
}

forma explícita tuvo un objetivo básicamente institucional "el restablecimiento de la unidad jerárquica»" como especifica Leibniz en un proyecto redactado en nombre de Molano, abad de Loccum. En este sentido fue un fracaso. No se llegó a tal reunificación de la cristiandad, ni siquiera dentro de los confines del Imperio que era el fin último de las conversaciones. Sí se logra abundar en la superación del clima de enfrentamiento que se establece en Alemania después de la paz de Westfalia. Además, hay consecuencias políticas colaterales como la elevación del ducado de Brunswick al noveno electorado en Alemania en 1692.

¿Qué es lo que dicho diálogo puede representar hoy? En un momento como el presente en el que se ha privatizado en Occidente la adhesión a una religión en sociedades que son plurales, la gestión de acuerdos diplomáticos con el asesoramiento de teólogos profesionales resulta tan ajeno a la sensibilidad prevalente en nuestra situación actual como los contextos de la Edad Media. La religión en sociedades con un relativamente alto nivel de educación se ha privatizado hasta el punto de que no se podría concebir una situación de esta naturaleza. Por ello, estas negociaciones no pueden constituir un precedente para una acción política que se ajusta al contexto actual.

¿Hasta que punto es posible utilizar la distinción entre creencias e ideas? Solo en la medida en que puede ayudar a entender determinados fenómenos en su momento. Lo hemos apuntado en el contexto de la redescripción de catolicidad y de humanidad, pero cada situación tiene sus propias exigencias y una buena explicación debe tenerlas en cuenta.

¿Hasta que punto es relevante utilizar el concepto de secularización? Es discutible por tres razones. En primer lugar habría que precisar lo que se entiende por una sociedad secularizada; En segundo lugar, hoy nos es mucho más factible pensar en un final de la historia que no sea secularizado como hace treinta años. En todo caso, la última página de nuestra historia no se ha escrito. Puede tener un valor explicativo dentro de un radio relativamente pequeño de tiempo, cuando se contrasta, por ejemplo, la forma de monarquía absoluta que emerge en el siglo XVII en Francia y el galicanismo que la corresponde con la concepción de política que acompaña la democracia en Francia en el siglo XIX donde en principio se prescinde completamente de una justificación teológica de los acontecimientos y de la autoridad. Tiene un valor indicativo en un radio relativamente corto, en una secuencia de acontecimientos, pero con el tiempo las sociedades cambian culturalmente en un grado que hace que las comparaciones sea imprecisas y necesiten un sin fin de matizaciones. En tercer lugar, ver la historia como teniendo un significado unívoco que se va revelando a través de los acontecimientos no solo es impreciso, sino conduce a devaluar períodos que se ven de transición. El esfuerzo del historiador es tratar de prestar a cada momento su propia envergadura. Cada sociedad acude a concepto de historia, a la filosofía y a la religión en su momento para encontrar un determinado equilibrio, y deben ser juzgados por la forma en que esto lo realizan. Las negociaciones irénicas son propias de un momento y por ello una buena expresión de las ambiciones y frustraciones de una época.

\footnotetext{
35 Leibniz 1869: 2-172.
} 


\section{LAS CONVERSACIONES Y LA FORMA DIALOGAL}

Hechas estas apreciaciones es fundamental aludir a lo que me parece más interesante desde el punto de formal de estas discusiones. Son formas de diálogo estilizado cuando el desarrollo de las ciencias sociales en los últimos decenios nos conducen a estar abierto al ámbito de la interlocución que se establece en una sociedad. Una de las funciones de la distinción entre ideas y creencias consiste justamente en permitir ese diálogo que no sólo exige conocimiento de un lenguaje, sino también de una cultura.

Por un lado hay que pensar en las conversaciones irénicas como una forma de diálogo donde los interlocutores toman posición los unos frente a los otros y buscan a través de la mutua interlocución crear algo así como un campo en el que se puede establecer la convergencia de sus posiciones y llegar a conclusiones que se concretan en un documento aceptable para todos los participantes. Detrás de las formas cortesanas usadas en la correspondencia se puede percibir la búsqueda de una forma de comunidad que sería compatible con las divergencias del punto de partida. Pero es interesante apuntar que este espacio de diálogo era improvisado, dependiendo mucho de las relaciones de confianza y de respeto de los interlocutores e incluso de la oportunidad política. Podemos apreciar como la tercera fase del primer dialogo entre católicos y protestantes, está lastrada por la desconfianza mutua entre Bossuet y Leibniz que solo en parte se logra superar ${ }^{36}$ Todo ello significa que pesaba el hecho de que no se contaba con una institucionalización previa de este espacio y en parte el fracaso se expresa en la ausencia de un contexto suficientemente consagrado. Volviendo a la noción de creencias, parece que se impone la puntualización de que la aparición de nuevas formas de religiosidad y las críticas contra la institución que las albergaba conduce a una conciencia de vacío y al sentimiento de desconcierto correlativo. Serán otras instituciones, la del estado, que pasarán a ocupar en parte ese vacío. Y por otro lado habrá un proceso de tolerancia y privatización del culto.

Es verdad que una necesidad experimentada como exigiendo el reforzamiento de una estructura de poder preponderante hubiera permitido probablemente que las conversaciones hubieran corrido mejor suerte. De hecho se nado con la corriente de la historia que hasta la revolución francesa, o incluso después ha consistido en reforzar la soberanía nacional al tratar de reforzar la vigencia de conceptos que implicaban en principio realidades supranacionales como las Iglesias. $Y$ es cierto que el auge del nacionalismo ha podido vivirse en algunos casos, como una religión secularizada. Pero el hecho es que la coyuntura forzó a una iniciativa que debía reforzar el poder del Imperio que, por su parte, estaba ya irremediablemente debilitado por la Reforma y que ponía de relieve la debilidad del Emperador. La forma de una negociación diplomática requería al tiempo un compromiso por parte de quienes participaban en las negociaciones, que realmente no se llegó a dar. Pero no había ni un aparato institucional previo ni la conciencia de la posibilidad de un poder análogo al que pronto sugerirá

\footnotetext{
36 Leibniz 1869: 2-383 y 2-390.
}

el Abate Saint Pierre y que el propio Leibniz no comentó de una manera demasiado favorable. ${ }^{37}$

También es importante entender que este espacio de diálogo requiere su propio tiempo para constituirse y encontrar su desarrollo. En principio tiene su tiempo de constitución que corre de manera paralela a la coyuntura política de la misma sociedad. Incide la última en la primera, pero se puede reconocer un atisbo de lógica interna en la medida en que se van desplegando las posiciones de los interlocutores, unos frente a otros.

En este sentido es relevante la aportación que Habermas he hecho a la noción de diálogo, pues los trabajos de éste se encuentran entre los que han sido de mayor influencia. Al no distinguir Leibniz, cómo podemos hacer nosotros, entre una razón instrumental y una razón comunicativa ${ }^{38}$, como propone Habermas, esta distinción sí bien es clara, tiene que matizarse. Por lo general la caracterización ética de la razón en Leibniz se centra en el conocimiento y dominio del universo ${ }^{39}$ y la consiguiente realización del individuo de acuerdo con referencias clásicas, pero es cierto que las iniciativas irénicas serían desde este punto de vista híbridas porque desde sus posiciones epistemológicas, resulta claro que Leibniz está buscando un acomodo con sus interlocutores que tenga un valor político. Se da una forma de entendimiento que permita el establecimiento de un consenso. El reconocimiento del interlocutor significa en el caso de Leibniz que no sólo son importantes las formas de educación mantenidas entre los interlocutores, ni la confianza nacida de un prolongado trato. También pesa una teoría de la perspectiva que entiende que sobre temas últimos caben, dada la limitación congénita del hombre, varios puntos de vista que pueden ser superados por una teología natural, pero que por otra parte son valiosas.

El proceso de dialogo irénico entendida como un proceso de interlocución, tiene una triple dimensión en la medida en que es una forma de incorporar el interlocutor a un nuevo ámbito de sociabilidad con quién en principio resulta un antagonista y debería convertirse en colaborador. Por otra parte en las argumentaciones figura de una manera destacada una búsqueda intelectual de formas y formulaciones que sean aceptables para todos interlocutores. Finalmente, es propio del proceso la configuración de un espacio respectivo entre los interlocutores que van definiéndose en el curso del diálogo.

El diálogo ideal de Habermas es muy distinto. ${ }^{40}$ Para empezar los interlocutores se encuentran siguiendo una razón que es ajena a los condicionamientos y perspectivas particulares. Lo fundamental en el diálogo irénico es, por el contrario, que su propia posición del interlocutor salga reforzada del diálogo. No es cualquier persona sino por el contrario debe tenerse en cuenta su formación, dignidad

37 Leibniz 1768: 5-56.

38 Aunque tal juicio debe matizarse teniendo en cuenta la distinción que utiliza Leibniz con la distinción entre Dios como arquitecto y Dios como monarca, no tiene esta distinción un peso importante en la configuración de su sistema. Por ejemplo en la Teodicea I 78 y 79. Leibniz 1885: 6-144 y ss. Cfr. también Salas 1994: 148 y ss.

39 Rossi 1966: 123 y ss. Toynbee 1993: 188 y ss. prestando más bien atención al mundo inglés, sin embargo, hace observaciones que son igualmente aplicables al continente.

40 Sigo fundamentalmente a Habermas 1984: 299 y ss. e ibídem 1999: 29 y ss. 
social y lealtades. En el caso del diálogo que defiende Habermas todo participante por el mero hecho de pertenecer a una sociedad evolucionada puede participar como miembro de pleno derecho. Por el contrario, los participantes en estos diálogos interconfesionales representan a las religiones a las que se han adherido, y por tanto a un poder institucionalmente establecido, y actúan por cuenta de otro. Acuden a la negociación con el animo de lograr un acuerdo que no les obligue a desdecirse de sus propios principios y al tiempo se ajuste a los intereses políticos que representan. Mientras que la opinión pública de Habermas tiene que hacer frente a una coyuntura incierta sin perjuicios previos, en el caso de estas negociaciones irénicas, aun dándose en una coyuntura que es igualmente incierta, pesa como una cuestión fundamental la coherencia con las lealtades ya establecidas. ${ }^{41}$ Se trata de reconocer al otro como hermano, no porque se ajusta a una moral racional, sino porque se trata de un acuerdo común e integral que respete la simetría de los interlocutores.

Ello determina que si bien nosotros adoptamos la posición de Leibniz que es la mejor conocida, las actuaciones de sus interlocutores son importantes por el hecho de que sus perspectivas se encuentran ya definidas. Incluso se puede llegar a contar la misma historia desde el punto de vista de Francisco de Rojas, Pellison, Madame de Brinon, la duquesa Sofia, la abadesa Luisa Hollandine, Bossuet, Molano, y Jablonski. Son puntos de vista distintos pero convergentes. Una de las importantes novedades de la última investigación sobre el tema son trabajos dedicados a Molano y Francisco de Rojas por un lado ${ }^{42}$ que cuentan la primera de las conversaciones asumiendo las diferencias entre los dos interlocutores. ${ }^{43}$

En este contexto si se desea mantener el concepto de una razón comunicativa - que se opondría a otros usos de la razón- tiene que hacerse sin acudir a la distinción kantiana entre un yo empírico y un yo racional, sino acudiendo a la noción leibniziana de percepción que teniendo dimensiones de negatividad, comprende el yo íntegro consciente e inconsciente a la vez, que se produce en su trato con el mundo. La razón comunicativa no sería el resultado de un uso "puro» de la razón, sino más bien se impone una lectura aristotélica pensando que Leibniz parte de una razón encarnada y, añadimos, socializada en una determinada perspectiva.

Fundamentalmente nos encontramos en un momento de transición que la parcial secularización de la cultura, a

41 En el caso de Leibniz ante sus interlocutores católicos se da frecuentemente la idea de que era un interlocutor cercano a realizar a convertirse al catolicismo. En realidad, en ese momento en el contexto del Imperio, y ante la división del protestantismo se daban frecuentes conversiones del bando protestante al catolicismo y no viceversa. El duque de Hannover, Juan Federico, que contrató a Leibniz en 1676 es un caso entre muchos que enumera Rommel en su edición de la correspondencia de Leibniz con el convertido al catolicismo duque de Hessen Rheinfels (Rommel 1847: 1-48). Su hermano por el contrario se mantuvo dentro del luteranismo de sus estados. Se estableció una relación de verdadera amistad entre los dos que da pie a una aclaración de su propia adhesión del propio Leibniz que en realidad sería acorde a su perspectivismo (A 1-4-320).

42 Masser 2002

${ }^{43}$ A estos trabajos se añaden la reciente edición de Jablonski y Leibniz 2013. En lo que respecta a Bossuet solo puedo reseñar la obra de Gaquère 1966. partir del siglo XIX, determina que el control de la religión pierde peso en una cultura. Pero eso no disminuye el interés de este episodio junto con otros que se pueden mencionar entre la Reforma y las grandes revoluciones de finales del siglo XVIII en los que una parte de la inteligencia europea trató de resolver el fraccionamiento de la cultura occidental que la Reforma había adquirido.

\section{BIBLIOGRAFÍA}

Antognazza, M.a R. 2007. Leibniz on the Trinity and the Incarnation. Reason and Revelation in the Seventeenth Century. Yale.

Backus, I. 2016. Leibniz, protestant theologian. Oxford.

Bossuet, J. B. 1848. Oeuvres Completes. Paris: Mellier Fréres. [Edición del Cardenal Bausset].

Duchhardt, H. 2015. "Leibniz und das 'Modell' des römisch-deutschen Reiches» en D. Beiderbeck, Umwelt und Weltgestaltuing. Leibniz's politisches Denken in seiner Zeit: 51. Göttingen.

Gaquère, F. 1966. Le dialogue Irénique Bossuet-Leibniz. La reunión des Eglises en échec (1691-1702). Paris.

Gilson, E. 2005. Les Métamorphoses de la cité de Dieu. Paris: Vrin.

Habermas, J. 1984. Teoría de la Acción Comunicativa: Complementos y estudios previos. Madrid: Cátedra.

Habermas, J. 1999. La Inclusión del Otro. Barcelona: Labor.

Jablonski, D. E. y Leibniz, G. W. 2013. Negotium Irenicum. L'union des Églises protestantes selon G. W. Leibniz et D. E. Jablonski. Paris: Garnier. [Edición de C. Rösler-Le Van].

Kelly, J. E. 2009. The Ecumenical Councils of the catholic Church. A History. Collegeville.

Leibniz, G. W. 1768. Opera Omnia. Ginebra. [Edición de L. Dutens Fratres de Tournes].

Leibniz, G. W. 1869. Oeuvres. Paris. [Edición de L. A. Foucher de Careil. Segunda edición. Firmin Didot frères].

Leibniz, G. W. 1885. Die Philosophischen Schriften. Berlin: Weidmannsche Buchhandlung. [Edición de C. I. Gerhardt].

Leibniz, G. W. 1923. Sämtliche Schriften und Briefe. Berlin: Brandenburgischen Akademie der Wissenschafften und der Akademie der Wissenschaften in Göttingen... Darmstadt y Berlin.

Leibniz, G. W. 1948. Textes Inédits. Paris. [Edición de G. Grua].

Masser, K. 2002. Cristóbal de Gentil de Rojas y Spinola O. F. M. und der lutherische Abt Gerardus Wolterius Molanus. Eine Beitrag zur Geschichte der Unionbestrebungen der katholischen und evangelischen Kirche im 17 Jahrhundert. Münster.

Miller, S. J. T. y Spielman J. 1962. "Cristóbal de Rojas y Spinola, cameralist and irenicist, 1625-1695». Transactions of the American Philosophical Society: New Series, V52, Part 5: 33 y ss.

Naert, E. 1968. "L'idée de religion naturelle selon Leibniz», en Leibniz. Aspects de l'homme et de l'oeuvre 1646-1716: 101. Paris: Aubier.

Neveux, J. 1968. "L'irénisme au temps de Leibniz et ses implications politiques", en Leibniz. Aspects de l'homme et de l'oeuvre 16461716: 69 y ss. Paris: Aubier.

Ortega y Gasset, J. 2006-2009-2010. Obras Completas. Madrid: Taurus. Rommel, Chr. von 1847. Leibniz und Landgraf Ernst von Hessen Rheinfels. Ein ungedruckter Briefwechsel ubre religiöse und politische Gegenstände. Frankfurt.

Rossi, P. 1966. Los filósofos y las maquinas 1400-1700. Barcelona: Labor.

Salas, J. de 1994. Razón y Legitimidad en Leibniz. Madrid: Tecnos.

Toynbee, A. 1993. La religion vue par un historien. Paris: Gallimard. 